\title{
Avaliação Psicológica no Século XXI: de Onde Viemos e para Onde Vamos
}

\author{
Ricardo Primi ${ }^{1}$ \\ ${ }^{1}$ Universidade São Francisco, SP, Brasil.
}

\begin{abstract}
Resumo: Na primeira parte, esse artigo fará um enquadramento amplo da avaliação psicológica dentre as atividades profissionais do psicólogo e uma breve descrição reflexiva histórica, dos últimos 18 anos, dos esforços empreendidos pelo Conselho Federal de Psicologia na tentativa de regulamentação da área, focando nas lições aprendidas. Na segunda parte, o artigo irá focar os eventos científicos e históricos ocorridos nos últimos cinco anos, especialmente no âmbito da inteligência artificial, os quais apontam para um novo papel da avaliação psicológica no mundo. O artigo faz uma reflexão sobre as implicações dessas mudanças para o papel do psicólogo e para a regulamentação de sua prática profissional.
\end{abstract}

Palavras-chave: Avaliação Psicológica, Aprendizagem Estatística.

\section{Psychological Assessment in the 21st Century: Where We Come from and Where We Go}

\begin{abstract}
In the first part, this article will provide a broad framework of psychological assessment of the psychologist's professional activities and a brief historical reflective description of the area's regulatory board efforts over the past eighteen years., focusing on the lessons learned. In the second part, the article will focus on scientific and historical events occurring in the last five years, especially in the field of artificial intelligence, which point to a new role of psychological assessment in the world. It presents a reflection on the implications of these changes for the role of psychologists and for the regulation of their professional practice.
\end{abstract}

Keywords: Psychological Evaluation, Statistical Learning.

\section{Evaluación Psicológica en el Siglo XXI: de Dónde Venimos y Hacia Dónde Vamos}

Resumen: En la primera parte, este artículo hará un encuadramiento amplio de la evaluación psicológica entre las actividades profesionales del psicólogo y una breve descripción reflexiva histórica de los esfuerzos del consejo de reglamentación del área en los últimos dieciocho años, enfocándose en las lecciones aprendidas. En la segunda parte, el artículo se centrará en eventos científicos e históricos ocurridos en los últimos cinco años, especialmente en el ámbito de la inteligencia artificial, que apuntan a un nuevo papel de la evaluación psicológica en el mundo. El artículo hace una reflexión sobre las implicaciones de estos cambios para el papel del psicólogo y para la regulación de su práctica profesional.

Palabras clave: Evaluación Psicológica, Aprendizaje Estadístico. 
Já se passaram 18 anos desde as primeiras iniciativas do Conselho Federal de Psicologia (CFP) para regulamentar a área de avaliação psicológica, as quais culminaram no Sistema de Avaliação dos Testes Psicológicos (Satepsi - http://satepsi.cfp.org.br). Esse tempo foi intensamente marcado por tensões, discussões e tentativas de construção de consensos na área. Pode-se citar vários avanços ocorridos nesse período, tais como a ampliação do número e qualidade dos instrumentos comercializados, a indução no aprimoramento do conteúdo da formação em avaliação, o estímulo, entre os profissionais, para a discussão sobre essa temática, a criação de consensos sobre os critérios científicos dos instrumentos (Miguel, 2014; Mihura, Meyer, Dumitrascu, \& Bombel, 2013; Primi, 2010; Villemor-Amaral, 2008). Pode-se concluir que o Satepsi foi um importante indutor de discussões e aprimoramento da área. Eventos recentes indicam a importância da avaliação psicológica para um universo mais amplo do que o trabalho tradicional do psicólogo no atendimento a pessoas, organizações e instituições. Tal ampliação requer uma reflexão sobre a prática profissional da avaliação psicológica. Em seguida esses tópicos serão abordados em mais detalhes, dividindo-se em: definição e regulamentação da avaliação psicológica, a origem do Satepsi, uso privativo dos testes, avaliação psicológica para além da Psicologia.

\section{Definição de avaliação psicológica}

A avaliação psicológica é entendida como um método sistemático de obter informações sobre o comportamento das pessoas, as quais serão usadas para inferir as suas características psicológicas. Ela ocorre em contextos delimitados com propósitos específicos, tais como: descrever as características das pessoas - por exemplo, em contextos clínicos nos quais as pessoas buscam autoconhecimento; predizer comportamentos futuros - por exemplo, em processos seletivos para achar pessoas que se sairão bem em um determinado trabalho; fazer classificação diagnóstica e planejamento de intervenções por exemplo, no contexto clínico, na busca do entendimento aprofundado de uma determinada queixa para se pensar no que poderia ser feito para a resolução do problema e redução do sofrimento psíquico; e monitorar variáveis psicológicas - por exemplo, no contexto educacional quando se quer monitorar o desenvolvimento cognitivo e/ou socioemocional das crianças e verificar o efeito global da educação nessas características (American Educational Research Association, American Psychological Association, \& National Council on Measurement in Education, 2014; Primi, \& Nunes, 2010).

Uma distinção importante é que a avaliação psicológica busca fazer inferências a respeito de um construto (inteligência, personalidade etc.). Um construto é um atributo das pessoas, não observável diretamente, que se postula existir e que se assume estar refletido nos comportamentos observados na testagem. Assim, é um conceito teórico sobre um atributo latente que explica os comportamentos na testagem (Cronbach, \& Meehl, 1955). Portanto, a prática da avaliação psicológica requer conhecimento especializado sobre as teorias psicológicas que irão definir esses construtos e também sobre psicometria, que irá especificar como instrumentos psicológicos são construídos para produzir inferências válidas sobre esses construtos latentes.

\section{Regulamentação brasileira sobre a avaliação psicológica}

A demanda desse conhecimento especializado faz com que a prática da avaliação psicológica seja precedida por um treinamento profissional no nível da graduação em Psicologia. Assim, essa prática foi regulamentada por uma Lei Federal de 1962, a qual afirma que "Constitui função privativa do Psicólogo a utilização de métodos e técnicas psicológicas com os seguintes objetivos: a) diagnóstico psicológico; b) orientação e seleção profissional; c) orientação psicopedagógica; d) solução de problemas de ajustamento", assim como determina que "É da competência do Psicólogo a colaboração em assuntos psicológicos ligados a outras ciências".

Essa lei enfatiza que o uso de instrumentos psicológicos para as finalidades indicadas é privativo aos profissionais da Psicologia. Também enfatiza que as relações interdisciplinares que envolvam conhecimentos da Psicologia devem ser feitas com a colaboração dos profissionais da Psicologia. Essa demanda está diretamente relacionada a formação profissional, a qual deve preparar os profissionais com as competências básicas para realizar a avaliação psicológica e uso adequado dos instrumentos de avaliação. Por exemplo, Noronha et al. (2002) descrevem que a formação profissional deve pelo menos cobrir os seguintes domínios: 
1) teoria da medida e psicometria; 2) avaliação da inteligência; 3) avaliação da personalidade, incluindo técnicas projetivas e os inventários de personalidade; 4) práticas integrativas de planejamento, execução e redação dos resultados da avaliação psicológica (elaboração de laudos) nos mais variados contextos, incluindo conhecimentos das mais diversas áreas da Psicologia (p. 174).

Mas também alertam que nem todos os cursos de Psicologia oferecem esses conteúdos.

\section{A origem do Satepsi: as queixas sobre os processos de avaliação}

Um dos principais problemas enfrentados pelo CFP, notados nas denúncias de infrações ao código de ética da Psicologia por parte de seus profissionais, refere-se à avaliação psicológica (Zaia, Oliveira, \& Nakano, 2018). Esse tipo de queixa por parte da sociedade em relação ao profissional é um tema historicamente recorrente (Frizzo, 2014). Em parte, isso ocorre porque a avaliação é um dos serviços mais frequentes oferecidos à pessoa comum. Muitas vezes ocorre em situações de alto impacto (high stake) com consequências sérias para as pessoas envolvidas, como por exemplo, na seleção de pessoal.

O Satepsi surgiu como reação a essa demanda. Em decorrência de limitações na formação, notava-se um problema crônico na seleção de instrumentos usados na prática profissional. Era notável, entre os instrumentos mais frequentemente utilizados em situação de alto impacto, figurarem alguns que careciam da fundamentação científica de embasamento para seu emprego (Noronha, Primi, \& Alchieri, 2004). Assim, o CFP decidiu criar um sistema de avaliação da qualidade dos instrumentos a partir de critérios científicos definidos pela área, os quais pudessem informar, aos profissionais, quais instrumentos teriam as condições mínimas para uso profissional. Assim surgiu o Satepsi.

Essa medida não impactou diretamente a origem do problema, que é a formação profissional em avaliação psicológica. Entretanto, indiretamente, mobilizou o CFP, os profissionais, pesquisadores e editoras ao redor dessa temática. Essa mobilização promoveu a qualificação da área em vários sentidos (Primi, 2010).

\section{O uso privativo de testes psicológicos: duas posições}

Uma discussão ocorrida na comissão consultiva do Satepsi e que ainda está viva no âmbito profissional é a questão do uso privativo dos testes psicológicos pelos psicólogos. Há uma tensão polarizada entre dois extremos: (a) de um lado, uma posição a favor do fechamento completo, adotada por profissionais/pesquisadores que defendem uma definição de teste psicológico abrangente e entendem que todos deveriam ser de uso privativo; e (b) do outro lado, uma posição favorável à abertura relativa, defendida por profissionais/pesquisadores que aceitam que alguns testes com características especiais poderiam ser de uso compartilhado com outras profissões específicas (medicina, educação, por exemplo). Nas discussões travadas sobre esse tema, a primeira posição sempre teve maioria entre os psicólogos.

Conforme discutido anteriormente, o uso privativo se sustenta na necessidade de conhecimento especializado para uso adequado dos instrumentos. Esse é um entendimento consensual entre todos os psicólogos, sobre o qual não há divergência. Mas o que ocorre, na prática, se um profissional de outra área tiver adquirido as competências necessárias para uso de algum instrumento específico tradicionalmente considerado como teste de avaliação psicológica restrito (uma bateria de avaliação da inteligência)? Imagine uma situação na qual tivéssemos uma formação em nível pós-graduação em neuropsicologia, oferecida a profissionais das áreas da saúde (psicólogos, fonoaudiólogos, médicos, enfermeiros etc.) e essa oferecesse formação teórica e metodológica necessária para uso de testes cognitivos em avaliação neuropsicológica. Isso não habilitaria esses profissionais, não psicólogos, a usarem esses instrumentos? Ainda, será que profissionais da psiquiatria não receberam formação teórica e metodológica adequada para uso de inventários e escalas de sintomas específicos (depressão e ansiedade) para realização de diagnóstico psiquiátrico? Esses e outros exemplos são um dos principais pontos que motivaram a posição de abertura relativa. Em contraponto, a posição a favor do fechamento completo entende que essas outras profissões não recebem formação suficientemente adequada para uso desses instrumentos. Além disso, entende que outros profissionais que precisam de algum tipo de informação obtida por meio de instrumentos de avaliação psicológica devem requisitar um psicólogo para fazê-lo, dentro de um trabalho de parceria em equipe multidisciplinar. 


\section{Psicologia e Fonoaudiologia: a primeira regulamentação a favor da abertura relativa}

É interessante mencionar que a abertura relativa já é uma realidade com a fonoaudiologia. O instrumento Neupsilin (Instrumento de Avaliação Neuropsicológica Breve, Salles et al., 2011) que é uma bateria de avaliação de processos cognitivos, e por isso considerado um teste psicológico, foi recentemente objeto de uma análise técnica conjunta entre os conselhos federais de Psicologia e Fonaudiologia. A conclusão dessas discussões envolveu o entendimento de que o Neupsilin pode ser usado por psicólogos e fonoaudiólogos para os propósitos específicos de cada especialidade (ver Resolução conjunta CFP/CFF n ${ }^{\circ}$ 01/2017).

$\mathrm{O}$ gatilho dessa medida foi um processo judicial pleiteado pelo Conselho Federal de Fonaudiologia (CFF) contra o CFP, argumentando que os fonoaudiólogos teriam competência necessária para uso do Neupsilin em suas práticas profissionais. Felizmente, a resolução desse conflito ocorreu, não por meio de uma batalha judicial entre os dois conselhos, mas sim pela formação de uma parceria que empreendeu diálogo e uma análise técnica que culminou em um entendimento consensual que é consonante com a posição favorável à abertura relativa. A lição aprendida nessa decisão é que, apesar de vigorar, no seio dos psicólogos, uma posição favorável ao fechamento completo, quando nos debruçamos em uma análise técnica dos fundamentos da limitação de uso de testes psicológicos, ela somente se sustenta em um modelo de restrição-por-competência e não pelo modelo atual de restrição-por-categoria-profissional atualmente em vigor na legislação do psicólogo.

É interessante notar que essa resolução abriu, pela primeira vez, um precedente de mudança para 0 modelo de restrição-por-competências no CFP. Se esse entendimento ocorreu em relação ao teste Neupsilin, por que não poderia ocorrer, por exemplo, com as escalas de depressão em relação aos médicos? Ainda, se os fonoaudiólogos podem usar o Neupsilin em sua prática de avaliação, porque não poderiam utilizar também outros instrumentos psicológicos que medissem construtos similares ao Neupsilin? Na prática, a Psicologia precisa se preparar para incorporar esse novo modelo em suas regulamentações profissionais de forma a evitar eventuais ações judiciais mais agressivas, as quais, talvez, não tenham um positivo quanto esse da Psicologia e Fonoaudiologia.

\section{A lei e a abertura relativa dos testes psicológicos}

Um detalhe importante da lei federal de 1962 que instaura o uso privativo de métodos e técnicas por psicólogos, isto é, por meio da implementação do modelo de restrição-por-categoria-profissional não é tão restritiva quanto a posição de fechamento completo gostaria.

A lei afirma, explicitamente, que a restrição aos psicólogos existe somente quando o instrumento for usado para uma das quatro finalidades (em psicodiagnóstico, Psicologia clínica, orientação psicoeducacional, seleção e orientação profissional). Assim, a lei não restringe o instrumento em si, mas o uso do instrumento nas finalidades especificadas. Existindo outras possibilidades de uso dos instrumentos psicológicos, além dessas quatro especificadas na lei, não há restrição de uso. Por exemplo, um médico que queira usar um teste psicológico de depressão para auxiliá-lo na finalidade de formular um diagnóstico psiquiátrico para fins de prescrição de medicamento, não poderia ser proibido, já que essa finalidade está fora dos âmbitos especificados na lei. A utilização isolada de um instrumento, nesse caso, não caracterizaria um processo mais amplo de diagnóstico psicológico e/ou avaliação psicológica. A única situação em que um instrumento se tornaria restrito ao psicólogo seria quando ele só pudesse ser usado para uma das quatro finalidades especificadas na lei.

Nesse mesmo sentido, em 2008, houve um parecer jurídico emitido sobre esse assunto pelo Conselho Regional de Medicina do Estado de São Paulo (CRM/ SP) em 2008 que afirma:

as limitações ao uso dos testes devem ser interpretadas de maneira restritiva, sendo que apenas os casos previstos em lei estão abarcados pela exclusividade do profissional psicólogo. A eventual utilização dos testes em pesquisas acadêmicas, por exemplo, ou com objetivos outros que não os descritos na lei, não são abrangidos pela restrição (http://www.cremesp.org.br, Parecer $n^{\circ} 61981$ ).

Esse documento explicita ainda que a restrição da comercialização dos testes aos psicólogos não tem respaldo na lei. 


\section{Abertura relativa não se contrapõe ao uso privativo por psicólogos}

Em suma, apesar da maioria dos profissionais da Psicologia terem um entendimento de que todos os testes psicológicos são restritos, isto é, terem um modelo de restrição-baseado-no- instrumento associado à posição de fechamento completo, a qualificação jurídica indica que já existe, na prática, uma abertura relativa. Tanto a Lei de 1962 já permitia o uso de testes psicológicos por outros profissionais em casos específicos, quanto, recentemente, o conselho passou a adotar essa posição no caso do uso do Neupsilin pela Fonoaudiologia.

Mas é importante ressaltar que a abertura existenteé limitada. Assim não se deve confundir a posição abertura relativa com uma posição extrema de abertura completa. Há casos claros que tipificam exercício ilegal da profissão de psicólogo. Por exemplo, quando administradores utilizam testes psicológicos para fazerem seleção de pessoal, ou médicos utilizam o Rorschach para realização de avaliação psicológica, tais práticas estão claramente previstas como atos privativos dos psicólogos.

Assim, a legislação atual sobre os testes psicológicos baseia-se em um modelo de restrição seletiva em razão da finalidade para os quais são usados - por categoria profissional. Os sistemas ideais de restrição de uso deveriam adotar algum modelo de restrição baseado em competências, tal como tem sido feito, por exemplo, nos Estados Unidos. Entretanto, em todas as discussões sobre esse assunto, nenhuma ideia viável de implementação de um modelo desse tipo foi proposta.

\section{Avaliação psicológica para além da Psicologia}

Na Figura 1 são enumerados possíveis usos com finalidades distintas das elencadas na lei e, portanto, situações nas quais os testes psicológicos potencialmente poderiam ser usados por outros profissionais não psicólogos. Esses exemplos não significam que outras pessoas fariam o papel do psicólogo, executando seu trabalho. Eles se referem a situações em que as ferramentas da Psicologia são úteis para objetivos diversos de outras áreas - como a educação, por exemplo - e, por isso, os psicólogos interagem com essas áreas em colaborações inter- e multiprofissionais provendo consultoria e oferecendo métodos de avaliação baseados em suas competências específicas. É um trabalho de oferecimento de conhecimento especializado sobre os métodos desenvolvidos na Psicologia (especialmente na psicometria) que, em última instância, resultará em algum processo de avaliação gerido por vários atores, não exclusivamente psicólogos, mas que precisa ser coordenado e supervisionado pelos psicólogos. Tarefas como administração dos testes e correção poderão ser executadas por técnicos treinados não psicólogos e muitas vezes são automatizadas como se verá adiante. Mas as interpretações dos resultados e uso da informação sempre irão requerer a consultoria de um psicólogo.

É interessante notar que a lei reforça a necessidade da participação do psicólogo nessas parcerias interdisciplinares quando indica que "É da competência do Psicólogo a colaboração em assuntos psicológicos ligados a outras ciências". Isso pode ser generalizado às situações que requerem uso dos instrumentos de avaliação psicológica para usos além dos tradicionais na clínica, escola e organizações.

\section{Avaliação psicológica e a agenda educacional da aprendizagem socioemocional}

Um exemplo recente que pode ser citado é o movimento da aprendizagem socioemocional no âmbito educacional, o qual expande os objetivos da educação formal, explicitando a inclusão de competências socioemocionais no currículo das escolas. A organização Collaborative for Academic, Social, and Emotional Learning (Casel - www.casel.org) define aprendizagem socioemocional como os processos e experiências que fomentam o desenvolvimento de conhecimentos, atitudes e habilidades necessárias para compreender e gerir emoções, definir e atingir metas positivas, ser empático, criar e manter relacionamentos positivos e tomar decisões responsáveis (Zins, \& Elias, 2007). Características socioemocionais foram também incluídas como competências gerais na Base Nacional Comum Curricular (Brasil, 2017).

Com a incorporação dessas características nos currículos, os sistemas de avaliação dos objetivos de aprendizagem incluirão novos instrumentos para monitorar seu desenvolvimento nas escolas. Essa tarefa faz uma demanda importante à Psicologia e suas técnicas e instrumentos de medida. Uma parte fundamental desses sistemas é o uso de instrumentos de avaliação válidos, precisos e viáveis para medida de características socioemocionais em larga escala (Primi, Santos, John, \& Fruyt, 2016; Santos, \& Primi, 2014). Mas a finalidade e objeto dessas avaliações são diferentes dos usos tradicionais dos testes psicológicos. O foco está na elaboração e ava- 


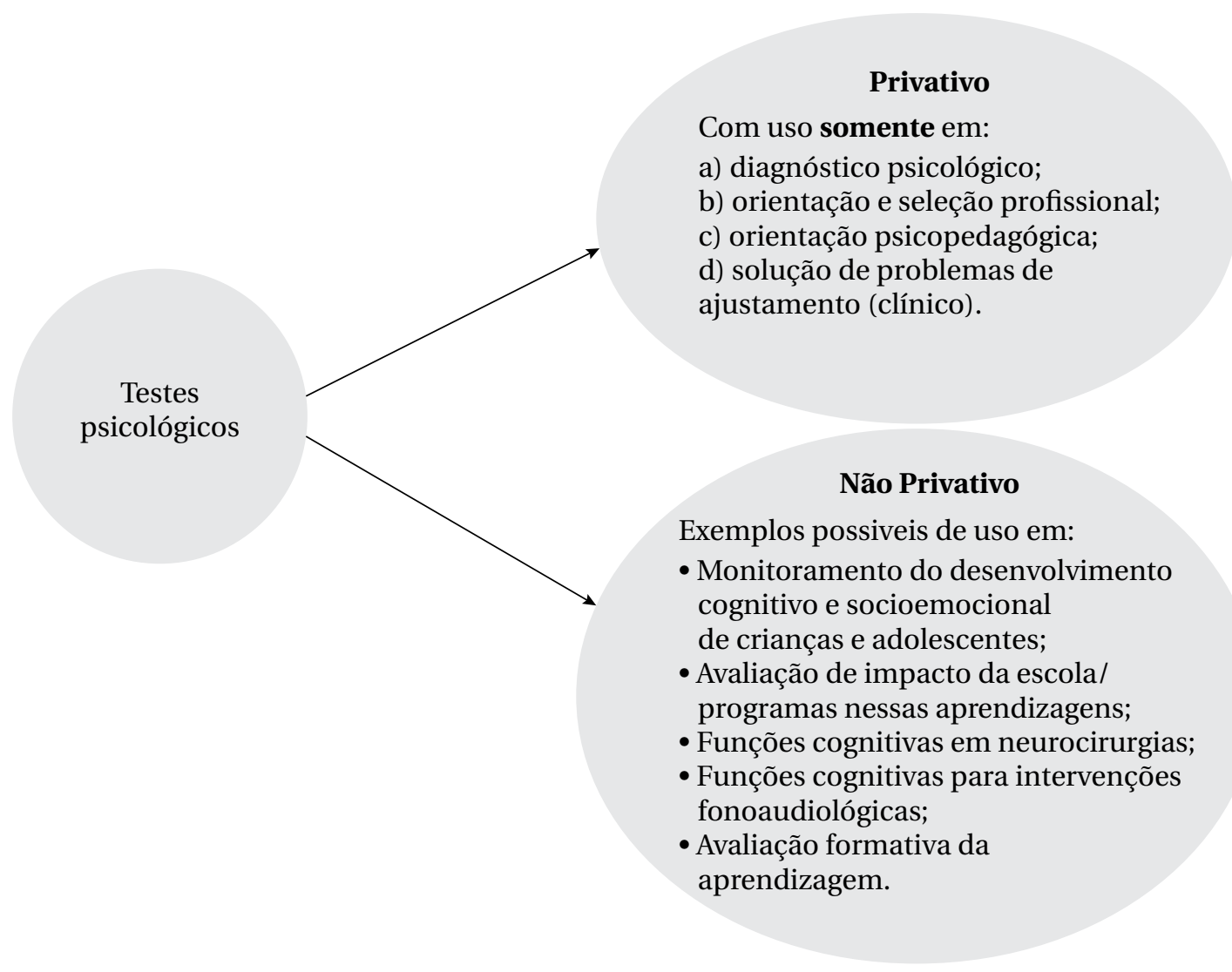

Figura 1

Exemplos de finalidades privativas (indicadas na Lei de 1962) e não privativas dos psicólogos.

liação de políticas públicas (grupos) e não na avaliação psicológica com vistas a orientações individuais intensivas. Busca-se monitorar as características psicológicas de forma geral, agregada por escolas, séries escolares, idade e outros grupos administrativos de interesse da gestão educacional, com vistas ao monitoramento do sistema educacional (ver, por exemplo, http://coredistricts.org/ our-work/school-quality-improvement-system/). Busca-se também avaliar impactos de intervenções educacionais nas características socioemocionais em estudos mais controlados. Os relatórios visam trazer informações úteis aos gestores (professores, diretores e secretários de educação, governos, entre outros) de forma que esses possam adaptar intervenções visando a melhoria do sistema. Em suma, nessas iniciativas, o uso que se dá aos instrumentos de avaliação em termos de propósito de uso e objeto de análise não se caracteriza como uma avaliação privativa que somente poderia ser administrada e controlada por psicólogos. Mas, evidentemente, requer o psicólogo como especialista em avaliação e medidas, oferecendo consultoria aos grupos interdisciplinares de atores envolvidos nesses grupos ajudando a interpretar os resultados desses instrumentos.

\section{Instrumentos de avaliação psicológica no PISA}

O Programme for International Student Assessment (PISA) produzido pela Organização para Cooperação e Desenvolvimento Econômico (OCDE) é aplicado no Brasil desde o ano de 2000 em ciclos de três anos avaliando Leitura, Matemática e Ciências de amostras de adolescentes de 15 anos, com o objetivo de descrever e comparar a qualidade da educação dos países participantes, visando aprofundar a análise dos sistemas educacionais a partir de uma comparação internacional (http://portal.inep.gov.br/web/ guest/pisa). Desde a primeira versão, o PISA inclui questionários contextuais que medem várias caraterísticas socioeconômicas, demográficas, e psicoeducacionais que permitem compreender contextos e processos associados à aprendizagem. Nos últimos 
sete ciclos, esses questionários incluíram escalas de avaliação de construtos psicológicos que apresentam validade preditiva do desempenho escolar, tais como: perseverança (conscienciosidade), abertura a experiências, integridade, senso de pertença à escola, entre outros (Lee, \& Stankov, 2018). Em 2021 incluirá provas de criatividade em dos módulos de provas cognitivas e questionários avaliando habilidades socioemocionais. Está em curso um estudo da OCDE que buscará compreender o desenvolvimento socioemocional de crianças e adolescentes em 11 países. Esse estudo incluirá um questionário de características socioemocionais baseado no modelo de personalidade dos Cinco Grande Fatores (Big Five), originado dos estudos taxonômicos da personalidade (Organization for Economic Cooperation and Development, 2017) ${ }^{1}$. Em suma, essas iniciativas exemplificam o uso expandido das teorias, técnicas e instrumentos desenvolvidos no âmbito da Psicologia demandado por outras ciências, quando estas precisam obter informações provenientes de variáveis psicológicas.

\section{Testagem psicológica sem testes na era digital}

Outro evento recente importante para essa discussão ocorreu nas eleições presidenciais americanas em 2016 que elegeram Donald Trump. Em 2013, Kosinski, Stillwell e Graepel da Universidade de Cambridge demostraram que características pessoais de usuários do Facebook, tais como traços de personalidade, preferências políticas, sexuais, entre outras, podiam ser previstas a partir do que as pessoas "curtiam" na rede (a partir de seus likes). Os autores aplicaram testes de personalidade, inteligência, coletaram informações sociais, culturais e demográficas, em uma amostra de 54.000 voluntários. Como esses usuários acessaram o aplicativo fazendo login a partir do Facebook, os pesquisadores conseguiram acesso aos dados de seu perfil. O Facebook armazena e fornece aos aplicativos comportamentos digitais dos usuários tais como: o que uma pessoa "curte" no Facebook, o que a pessoa posta na rede, dentre outras informações ${ }^{2}$. Com base nesses dados, utilizaram técnicas estatísticas de agrupamento de dados (single value decomposition, que é similar à análise de componentes principais) e aprendizagem de máquina (machine learning) para predizer características de personalidade a partir dos likes.

Os autores demostram que os escores baseados nos likes tinha forte correlação com traços de personalidade dos sujeitos (extroversão $r=0,40$; abertura $r$ $=0,43$; agradabilidade $r=0,30$; conscienciosidade $r=$ 0,29 ; e estabilidade emocional $r=0,30$ ) e outras características pessoais $(65 \%$ de classificações corretas em relação ao uso de drogas e $85 \%$ na classificação entre democratas versus republicanos) ${ }^{3}$. Os autores concluíram o artigo trazendo uma discussão do dilema ético desse achado já que, embora o usuário do Facebook tenha controle e consinta, explicitamente, o que torna público em seu perfil, ele não tem consciência de que, indiretamente, pode deixar rastros de suas características pessoais escondidas em informações aparentemente inofensivas, como seus likes.

Alguns anos depois, Aleksandr Kogan, também da Universidade de Cambridge, mas de um grupo independente sem conexão com Michal Kosinski, construiu um aplicativo via Facebook chamado thisisyourdigitallife que coletava dados similares a Kosinski (personalidade e likes do Facebook). Nessa época, se um usuário efetuasse login com o usuário do Facebook, o aplicativo tinha acesso aos dados que essa pessoa autorizava ser divulgado e também de todos seus amigos. Rapidamente esse aplicativo armazenou dados de mais de 80 milhões de pessoas (a maioria dos Estados Unidos). Kogan compartilhou essa base de dados com uma empresa de marketing político chamada Cambridge Analytica (https://cambridgeanalytica.org) ${ }^{4}$.

Nessa base cerca de 400 mil pessoas tinham respondido a um teste de personalidade e aproximadamente 80 milhões tinham seus dados do Facebook fornecidos indiretamente. Com isso foi possível construir um modelo preditivo, usando as técnicas demonstradas por Kosinski et al. (2013) que observavam os likes e previam os escores nos cinco fatores do big five (John, Naumann, \& Soto, 2008). Esse modelo foi aplicado usando os likes do restante da amostra para se obter uma estimativa dos escores no big five para cada pes-

\footnotetext{
${ }^{1}$ https://codeactsineducation.wordpress.com/2018/01/16/pisa-for-personality-testing/

${ }^{2}$ Evidentemente a disponibilidade dessas informações dependem da configuração dos ajustes de privacidade controlados pelo usuário. ${ }^{3}$ Os autores colocaram esse recurso disponível em https://applymagicsauce.com. É possível obter uma previsão de suas características pessoais a partir do seu perfil fazendo o login com o Facebook.

${ }^{4}$ O evento envolvendo a Cambridge Analytica foi tão importante que mereceu uma entrada na Wikipedia: https://en.wikipedia.org/wiki/ Cambridge_Analytica.
} 
soa da base. Com isso Cambridge Analytica obteve o perfil predito para as 80 milhões de pessoas na base.

Em 2016 a campanha de Donald Trump contratou os serviços da Cambridge Analytica para fazer seu marketing político. Tendo obtido a informação do perfil psicológico e unindo a outras informações como a localização dessa população imensa de americanos, seus hábitos de consumo entre outros. Ela mapeou o perfil psicológico no big five das regiões eleitorais americanas ${ }^{5}$. Baseados nesse perfil, criaram uma campanha de marketing usando micro-targeting $^{6}$ tentando influenciar a decisão de votos dos eleitores. Essa técnica procura adaptar cada mensagem, baseando-se na informação da personalidade dos receptores e endereçando propagandas específicas adaptadas a cada tipo de personalidade. Com isso se tem um poder muito mais forte de influência (Matz, Kosinski, Nave, \& Stillwell, 2017).

É muito difícil saber se, de fato, essa ação teve impacto na eleição americana. Mas há um consenso que é uma técnica de persuasão poderosa, muito mais avançada do que as práticas anteriores. Uma das principais discussões decorrente desse evento foi em relação à dimensão ética. Os voluntários de Aleksandr Kogan participaram da pesquisa com o objetivo de receberem informações sobre seu perfil de personalidade. Mas não sabiam que suas informações poderiam ser usadas em uma campanha de marketing. Menos consciência ainda tinham os amigos desses voluntários, de que seus perfis haviam sido usados subsequentemente para filtrar seletivamente os anúncios que veriam no Facebook com vistas a gerar simpatia de uma causa política. Ainda que os usuários do Facebook saibam que seus dados possam ser usados por aplicativos para customizar sua experiência, o tipo exato do uso fica nebuloso. Especialmente nesse evento se soubessem do uso para campanha política talvez tivessem restringido a autorização de uso de seus dados.

\section{Avaliação psicológica: back to the future}

$\mathrm{O}$ que esse evento nos indica sobre a natureza e usos da avaliação psicológica? Os comportamentos digitais refletem as características pessoais do agente, isto é, variáveis latentes representando os traços de personalidade, interesse ou inteligência, por exemplo. Assim, os mesmos princípios psicométricos dos modelos reflexivos estão na base da técnica usada. Isto é, esse método faz a agregação de indicadores - comportamentos digitais - para inferir características latentes - traços de personalidade. A diferença em relação aos testes psicológicos tradicionais está nos indicadores e nas técnicas de agregação. Nesses sistemas automatizados, os indicadores ou o comportamento digital, é armazenado constantemente pelas grandes empresas, gerando-se imenso banco de dados com comportamentos digitais dos usuários. Assim se tornam um banco potencialmente rico de indicadores para construtos psicológicos e outros aspectos do comportamento como saúde, padrões de consumo etc. No caso do Facebook dois tipos de dados já foram testados com relativo sucesso como indicadores de traços de personalidade e inteligência: os likes e as postagens (Kosinski et al., 2013; Park et al., 2015).

A metodologia de agregação é outra diferença. Um dos desafios, nesse caso, tem relação com a estrutura complexa e peculiar dos dados. Por exemplo, no estudo mencionado sobre os likes (Kosinsky et al. 2013) os autores tinham uma matriz de 58 mil linhas, uma para cada usuário, e 55.814 colunas uma para cada like. Grande parte das células usuário-like era vazia. Essas colunas foram reduzidas a 100 dimensões usando uma análise similar à dos componentes principais e depois se utilizou regressão com procedimentos de aprendizagem de máquina para se chegar a um modelo preditivo dos traços de personalidade a partir dos likes?.

Em síntese, esses métodos inovadores, inicialmente desenvolvidos no Laboratório de Psicometria de Cambridge consistem em adaptações de técnicas psicométricas para esse tipo de dado específico disponível atualmente nas redes sociais, com vistas à criação de instrumentos psicológicos a partir da análise do comportamento digital. O racional desse procedimento se assemelha ao método de criação de gabarito empírico usado nos testes: Inventário Multifásico Minesota de Personalidade (MMPI) e o teste de interesse profissional de Strong. Esse procedimento descobre o esquema de pontuação dos indicadores de forma empírica para

\footnotetext{
${ }^{5}$ theguardian.com/technology/2018/mar/17/facebook-cambridge-analytica-kogan-data-algorithm

${ }_{6}$ https://www.firstpost.com/tech/news-analysis/big-data-and-psychographic-profiling-helped-donald-trump-win-the-us-presidential-election-3696847.html

${ }^{7} \mathrm{~A}$ amostra foi dividida em 10 subgrupos com o mesmo número de pessoas para se derivar o modelo em uma amostra e testar sua eficácia em outra.
} 
maximizar a previsão do critério externo (Cronbach, 1996). No caso do MMPI, o critério externo era a pertença as categorias diagnósticas psiquiátricas da época e, no Strong, as profissões. Assim esse estudo ressuscitou um método antigo de gabarito empírico atualizando-o e aprimorando-o com as tecnologias inovadoras atuais da ciência da computação.

Isso ocorre em razão de uma grande revolução que está em curso no campo mais amplo da inteligência artificial. Os métodos de inteligência artificial como deep learning, machine learning, procuram automaticamente descobrir padrões nos dados, criar representações úteis que permitam a previsão de alguma variável relevante (nos exemplos citados os traços autorrelatados de personalidade). Nos últimos cinco anos houve um grande avanço nos problemas aplicados, de modo que esses métodos conseguem atingir resultados similares ou melhores que o desempenho humano. Veja, por exemplo, os avanços nas áreas de reconhecimento de imagens, de voz, tradução de textos, entre outros (Chollet, \& Allaire, 2018). A avaliação psicológica não foi uma exceção. Na psicometria essa metodologia tem seus fundamentos em um princípio antigo, mas transformado pelas técnicas atuais mais avançadas da inteligência artificial e a disponibilidade de grandes bases de dados de comportamentos digitais.

Como se caracteriza a avaliação psicológica nesse caso? O que é teste psicológico nesse caso? No exemplo citado, o teste é o modelo preditivo que foi criado de forma automática configurando-se em uma representação dos likes que consegue predizer traços de personalidade. Mas não há explicação teórica alguma sobre porque os indicadores refletem o construto, como ocorre no desenvolvimento tradicional de um teste psicológico. Ainda assim, o sistema procura prever os traços de personalidade obtidos por meio de um instrumento de avaliação psicológica. Assim, dependendo do propósito para o qual esse instrumento for usado ele poderá ser considerado de uso privativo na legislação brasileira pelas mesmas razões que foram elencadas anteriormente. Entretanto, esses sistemas são criados com propósitos muito amplos, diferentes dos quatro especificados na lei, como o marketing político. Assim, eles exemplificam uma contribuição do conhecimento aplicado da psicometria e seus métodos de avaliação em um âmbito inter e multiprofissional. Ressalta-se, no entanto, que é fundamental a participação do psicólogo para dar sentido as informações obtidas desses instrumentos automatizados já que se tratam de perfis psicológicos dos usuários.

\section{Considerações finais}

Nos últimos quinze anos houve uma grande evolução na área da avaliação psicológica fomentada pelo Satepsi. Ao mesmo tempo, as demandas de outras áreas têm ressaltado a importância central das ferramentas psicológicas para a sociedade, como nos exemplos citados da avaliação educacional e no uso em campanhas de marketing - ainda que esse último tenha sido um mau exemplo em termos de conduta ética do uso das informações decorrentes da avaliação. O que esses eventos indicam é a importância essencial que a Psicologia em geral e a psicometria, em particular, possuem em problemas mais amplos enfrentados por outras disciplinas. Também indicam a responsabilidade decorrente de quais usos implicam no fomento do uso adequado de suas técnicas nesse âmbito mais amplo. Conforme se demonstrou, os instrumentos de avaliação já ultrapassaram os limites do consultório e controle exclusivo do psicólogo. Os instrumentos já são compartilhados com outras ciências em propósitos mais amplos que vão além do trabalho mais tradicional do psicólogo. Assim é necessária uma reflexão e construção de orientações de boas práticas da Psicologia na sua relação com outras ciências. Especialmente porque o trabalho do psicólogo nesse âmbito passa a ser cada vez mais interdisciplinar, requerendo desse, que contribua explicando os fundamentos de seus métodos e seus resultados nas aplicações em problemas diversos de outras áreas. Talvez esse seja um dos principais desafios do Satepsi para o século XXI.

\section{Referências}

American Educational Research Association, American Psychological Association, \& National Council on Measurement in Education (Eds.). (2014). Standards for educational and psychological testing. Washington, DC: American Educational Research Association. Recuperado de http://www.apa.org/science/programs/testing/standards.aspx

Brasil. Ministério da Educação. (2017). Base Nacional Comum Curricular (BNCC). Brasília, DF: o autor. Recuperado de http://portal.mec.gov.br/conselho-nacional-de-educacao/base-nacional-comum-curricular-bncc 
Chollet, F., \& Allaire, J. J. (2018). Deep Learning with R. New York, NY: Manning.

Cronbach, J. L. (1996). Fundamentos da testagem psicologica. Porto Alegre, RS: ArtMed.

Cronbach, L. J., \& Meehl, P. E. (1955). Construct validity in psychological tests. Psychological Bulletin, 52(4), 281302. https://doi.org/10.1037/h0040957

Frizzo, N. P. (2014). Infrações éticas, formação e exercício profissional em psicologia. (Dissertação). Universidade Federal de Santa Catarina, Florianópolis, SC.

John, O. P., Naumann, L. P., \& Soto, C. J. (2008). Paradigm shift to the integrative Big Five trait taxonomy: History, measurement, and conceptual issues. In O. P. John, R. W. Robins, \& L. A. Pervin (Eds.), Handbook of personality: Theory and research (pp. 114-158). New York, NY: Guilford.

Kosinski, M., Stillwell, D., \& Graepel, T. (2013). Private traits and attributes are predictable from digital records of human behavior. Proceedings of the National Academy of Sciences, 110(15), 5802-5805. https://doi.org/10.1073/ pnas. 1218772110

Lee, J., \& Stankov, L. (2018). Non-cognitive predictors of academic achievement: Evidence from TIMSS and PISA. Learning and Individual Differences, 65, 50-64. https://doi.org/10.1016/j.lindif.2018.05.009

Matz, S. C., Kosinski, M., Nave, G., \& Stillwell, D. J. (2017). Psychological targeting as an effective approach to digital mass persuasion. Proceedings of the National Academy of Sciences, 114(48), 12714-12719. https://doi.org/10.1073/ pnas.1710966114

Miguel, F. K. (2014). Myths and facts in teaching of projective techniques. Psico-USF, 19(1), 97-106. https://doi. org/10.1590/S1413-82712014000100010

Mihura, J. L., Meyer, G. J., Dumitrascu, N., \& Bombel, G. (2013). The validity of individual Rorschach variables: systematic reviews and meta-analyses of the comprehensive system. Psychological Bulletin, 139(3), 548-605. https://doi.org/10.1037/a0029406

Noronha, A. P. P., Ziviani, C., Hutz, C. S., Bandeira, D., Custódio, E. M., Alves, I. B. et al. (2002). Em defesa da avaliação psicológica. Avaliação Psicológica, 1(2), 173-174.

Noronha, A. P., Primi, R., \& Alchieri, J. C. (2004). Parâmetros psicométricos: uma análise de testes psicológicos comercializados no Brasil. Psicologia: Ciência e Profissão, 24(4), 88-99. https://doi.org/10.1590/S141498932004000400011

Organization for Economic Cooperation and Development - OECD. (2017). Social and emotional skills well-being, connectedness and success. Paris: o autor. Recuperado de http://www.oecd.org/education/school/UPDATED\%20 Social\%20and\%20Emotional\%20Skills\%20-\%20Well-being,\%20connectedness\%20and\%20success.pdf\%20 (website).pdf

Park, G., Schwartz, H. A., Eichstaedt, J. C., Kern, M. L., Kosinski, M., Stillwell, D. J. et al. (2015). Automatic personality assessment through social media language. Journal of Personality and Social Psychology, 108(6), 934-952. https:// doi.org/10.1037/pspp0000020

Primi, R. (2010). Avaliação psicológica no Brasil: Fundamentos, situação atual e direções para o futuro. Psicologia: Teoria e Pesquisa, 26(n.esp.), 25-36. https://doi.org/10.1590/S0102-37722010000500003

Primi, R., \& Nunes, C. H. S. S. (2010). O SATEPSI: propostas de aprimoramento. In: Conselho Federal de Psicologia-CFP. (Org.), Avaliação psicológica: Diretrizes na regulamentação da profissão (Vol. 1, pp. 129-148). Brasília, DF: o autor.

Primi, R., Santos, D., John, O. P., \& Fruyt, F. D. (2016). Development of an inventory assessing social and emotional skills in Brazilian youth. European Journal of Psychological Assessment, 32(1), 5-16. https://doi.org/10.1027/1015-5759/a000343

Resolução Conjunta №. 01/2017. Institui conjuntamente regras para a utilização da Coleção NEUPSILIN (Instrumento de Avaliação Neuropsicológica Breve NEUPSILIN e Instrumento de Avaliação Neuropsicológica Breve Infantil NEUPSILINInf) para uso exclusivo dos/as Fonoaudiólogos/as e Psicólogos/as. Recuperado de http://www.fonoaudiologia.org.br/ cffa/wp-content/uploads/2013/07/res-conjunta-neupsilim.pdf

Salles, J. F., Fonseca, R. P., Rodrigues, C. C., Mello, C. B., Barbosa, T., \& Miranda, M. C. (2011). Desenvolvimento do instrumento de avaliação neuropsicológica breve infantil NEUPSILIN-INF. Psico-USF, 16(3), 297-305.

Santos, D., \& Primi, R. (2014). Desenvolvimento socioemocional e aprendizado escolar: uma proposta de mensuração para apoiar políticas públicas. São Paulo: Instituto Ayrton Senna. 
Villemor-Amaral, A. E. (2008). A validade teórica em avaliação psicológica. Psicologia: Ciência e Profissão, 28(1), 98-109. https://doi.org/10.1590/S1414-98932008000100008

Zaia, P., Oliveira, K.S., \& Nakano, T.C. (2018). Análise dos processos éticos publicados no Jornal do Conselho Federal de Psicologia. Psicologia: Ciência e Profissão, 38(1), 8-21. https:// doi.org/10.1590/1982-3703003532016

Zins, J. E., \& Elias, M. J. (2007). Social and Emotional learning: promoting the development of all students. Journal of Educational and Psychological Consultation, 17(2-3), 233-255. https://doi.org/10.1080/10474410701413152

\section{Ricardo Primi}

Programa de Pós Graduação Stricto Sensu em Psicologia, Universidade São Francisco., SP, Brasil.

E-mail: rprimi@mac.com

O autor recebe financiamento para suas pesquisas do CNPq e do Instituto Ayrton Senna.

Endereço para envio de correspondência:

Universidade São Francisco - Programa de Pós-graduação em Psicologia.

Rua Waldemar César de Silveira, 105 - Swift - CEP: 13045-510.

Campinas - SP. Brasil.

Recebido $30 / 07 / 2018$

Aprovado 08/08/2018

Received $07 / 30 / 2018$

Approved 08/08/2018

Recibido 30/07/2018

Aceptado 08/08/2018

Como citar: Primi, R. (2018). Avaliação psicológica no Século XXI: De onde onde viemos e para onde vamos.

Psicologia: Ciência e Profissão, 38(n.spe), 87-97. https://doi.org/10.1590/1982-3703000209814

How to cite: Primi, R. (2018). Psychological Assessment in the 21st Century: Where We Come from and Where We Go. Psicologia: Ciência e Profissão, 38(n.spe), 87-97. https://doi.org/10.1590/1982-3703000209814

Cómo citar: Primi, R. (2018). Evaluación psicológica en el Siglo XXI: De dónde venimos y hacia dónde vamos . Psicologia: Ciência e Profissão, 38(n.spe), 87-97. https://doi.org/10.1590/1982-3703000209814 